This item is the archived peer-reviewed author-version of:

A murine model of lung ischemia and reperfusion injury : tricks of the trade

\title{
Reference:
}

Gielis Jan, Jungraithmayr Wolfgang, Boulet Gaëlle A.V., Bogers Johannes J.P.M., Weder Walter, Cos Paul, van Schil Paul.- A murine model of lung ischemia and reperfusion injury : tricks of the trade

Journal of surgical research - ISSN 0022-4804 - (2014), p. 1-8

DOI: http://dx.doi.org/doi:10.1016/j.jss.2014.12.008 


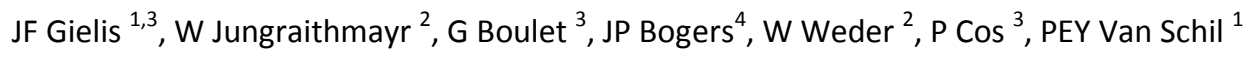

\footnotetext{
${ }^{1}$ Dept. of Thoracic and Vascular Surgery, Antwerp University Hospital, Antwerp, Belgium

${ }^{2}$ Dept. of Transplantation Surgery, Zürich University Hospital, Zürich, Switzerland

${ }^{3}$ Laboratory for Microbiology, Parasitology and Hygiene, Antwerp University, Antwerp, Belgium

${ }^{4}$ Applied Molecular Biology Research group, University of Antwerp, Antwerp, Belgium
}

\section{A murine model of lung ischemia and reperfusion injury: tricks}

\section{of the trade}

Author contributions:

Study conception and design: GIELIS - JUNGRAITHMAYR - COS - VAN SCHIL

Acquisition of data: GIELIS - JUNGRAITHMAYR - BOGERS

Analysis and interpretation of data: GIELIS - JUNGRAITHMAYR - BOULET

Drafting of manuscript: GIELIS - BOULET

Critical revision: WEDER - COS - VAN SCHIL 


\section{Abstract}

\section{Introduction}

Pulmonary ischemia-reperfusion injury (IRI) causes post-operative morbidity in patients undergoing lung transplantation, isolated lung perfusion and cardiopulmonary bypass and may lead to potentially lethal pathologies such as respiratory shock. In-depth study of this pathology requires a reliable animal model. Mice are a popular species to develop experimental models because of their logistic advantages and the availability of knock-outs. However, their small size warrants microsurgical techniques and a skilled surgeon.

\section{Materials \& Methods}

We developed a murine model of pulmonary anoxic IRI through hilar clamping using adult female Swiss mice. After left thoracotomy, we expose the pulmonary hilum keeping the ribs and the muscles of back and forepaw intact. A microvascular clamp is placed over the entire hilum, occluding bronchus, pulmonary artery and vein.

\section{Results}

Our model proved to be simple, reliable and reproducible. It showed minimal per- and postoperative mortality. Histopathological analysis indicated all characteristic features of pulmonary IRI, such as an | early recruitment of lymphfocytes followed by neutrophil influx.

\section{Conclusion}

This paper presents a murine surgery model for pulmonary IRI based on a muscle-sparing thoracotomy. The minimal approach limits manipulation of lung tissue, minimizing mortality and non-IRI-induced injury.

Keywords: pulmonary ischemia-reperfusion; ischemia-reperfusion injury; mouse; experimental model

\section{Number of words: 4220}




\section{Introduction}

Pulmonary ischemia and reperfusion occurs when both ventilation and blood flow to the pulmonary tissue are temporarily cut off. This triggers numerous many different intra- and extracellular physiological responses such as increased vascular permeability and pulmonary edema, immune system activation with lymphocyte and neutrophil infiltration, a hypercoagulable state ${ }_{2}$ and the induction of numerous cell signaling pathways(1).(1) The severity of the resulting cell and tissue damage is related to the duration of ischemia time and reperfusion time. The overwhelming inflammatory process, which is called ischemia reperfusion injury (IRI), presents an important complication of organ transplantation, a procedure that by definition involves complete organ anoxia(2).(2) Within 72 hours after pulmonary transplantation, reperfusion of a transplanted lung may cause alveolar damage, pulmonary edema and hypoxemia. Over $15 \%$ of transplant patients suffer from IRI-related graft complications in the immediate post-transplantation period(3).(3) Severe IRI reduces graft longevity, increases the risk of future chronic graft rejection and may ultimately result in primary graft dysfunction (PGD). The pathophysiological pattern of PGD resembles acute respiratory distress syndrome (ARDS) with severe hypoxemia, lung edema and diffuse alveolar damage and infiltrates. Itand is associated with a 30-day mortality of up to 50\%(4).(4) PGD, like ARDS, involves non-cardiogenic pulmonary edema that results in respiratory shock due to severely dysfunctional gas exchange. It is the major cause of immediate post-operative morbidity and mortality after lung transplantation. PGD is also an important determinant of the later occurrence of chronic pulmonary graft rejection, or bronchiolitis obliterans (BO) syndrome. BO is characterized by airway lumen obliteration and progressive fibrosis of the lung. It is the major cause of late mortality after lung transplantation. Pulmonary IRI is not only a transplantation-related complication, but may also occur after cardiac bypass surgery, pulmonary embolism or bronchial sleeve resection. 
Numerous research studies investigate the pathogenesis of IRI in different organs or focus on methods to tackle its detrimental effects. High-quality animal models play a crucial role in the translation of in vitro experimental findings to the patient bedside. Mice provide attractive experimental animal models with regard to economic and logistical factors, but are particularly interesting because of the broad availability of transgenic strains and genetic knock-outs. However, their small size complicates surgical procedures, which are almost by definition microsurgical procedures, and require both a skilled hand and a good protocol to ensure optimal results with minimal complications and variability that may confound the experimental data.

This paper provides a detailed description of the surgical procedure to construct a murine model of pulmonary anoxic IRI based on hilar clamping after a left muscle-sparing thoracotomy.

It is a model of warm ischemia-reperfusion injury, in which the ischemic lung is not removed from the body. A model of cold ischemia-reperfusion injury would imply the complete resection of the lung, temporary storage of the transplant in a cold buffer solution and subsequent re-implantation as described previously by Jungraithmayr et al. [REF Transplantation]. The transplant model is a very advanced microsurgical procedure, which is more difficult to learn compared to the model discussed here. Our procedure may serve as a 'step-up' toward more complicated thoracic procedures in mice. To our knowledge, there is currently no literature available that addresses the difference in effect of warm versus cold ischemia on the pathophysiological outcome of the ischemic and reperfused murine lung. A study in rabbits revealed that cooling of the ischemic lung in situ greatly reduced reperfusion injury, without siginificant differences compared to baseline level in alveolar edema, neutrophil infiltration or oxygenation level. Another porcine study indicated that a relatively short ( 90 minutes) period of warm ischemia is equivalent to the pathophysiologic changes that occur after considerably longer (24h) periods of cold ischemia. This implies that the model of warm IR described here can possibly serve as an accelerated model of cold IR, adequately inducing the same pathophysiological changes, only in a shorter 
timeframe. However, this hypothesis still has to be verified in a murine model of cold vs. warm ischemia.

The technique we present is also valuable to evaluate the effect of warm ischemia on reperfusion injury,

as in pulmonary emboli or trauma surgery. In order to compare this to the effect of cold ischemia, one

should indeed master the pulmonary transplantation technique as described previously.

-In this paper, wWe present a complete overview of the required materials, identify potential pitfalls and troubleshoot commonly encountered difficulties. The step-by-step illustrations support any aspiring mouse surgeon to perform a successful procedure. Our approach results in a reliable murine model of pulmonary anoxic IRI, while minimizing the variability of the experimental data, animal mortality, discomfort

and

possible

confounding

factors. 


\section{Microsurgical method}

\section{Preparation}

\section{Animal strain}

In general, any of the common strains of laboratory mice (Swiss Webster, C56BL/6 and BALB/c) can be subjected to hilar clamping using the procedure described in this paper. We have performed the procedure on these three strains.

Swiss Webster outbred mice provide an attractive strain for experimental surgery due to their low cost and gentle character. Some reports suggest that Swiss mice are less prone to acute kidney IRI compared to inbred strains, such as BALB/C or $\mathrm{C} 57 \mathrm{BL} / 6$ mice(5). (5) However, when reperfusion times of less than

24 hours are maintained, the amount of IR-related damage to the kidney seems to be equivalent among the different strains. Because of their slightly larger size than C57bl/6 and BALB/c mice, Swiss Webster mice are very useful to train beginners. A non-experienced surgeon should learn every step of the described procedure on a deceased animal before operating on living animals.

C57BL/6 mice, an inbred strain with minimal genetic variability between individual animals, tend to be more aggressive and expensive than Swiss Webster mice. The strain is commonly used in gene transfer and knockout models and therefore preferred for many experimental procedures. However, since C57BL/6 mice are smaller than Swiss Webster mice, surgical procedures become more challenging. Our preliminary data suggest that influx of inflammatory cells during pulmonary IRI may be more elaborate in Swiss mice compared to C57BL/6 mice (table 2). There is also some evidence suggesting that C57BL/6 mice tolerate myocardial IRI better, compared to Swiss mice, although more research on the effect of animal strain on IRI should definitely be undertaken_(6). 
$B A L B / C$ mice, another inbred strain, have extensive connective and fatty tissue around heart and hilum that complicates thoracic surgery, since it obscures the surgical field. Placing the clamp in a correct and reproducible way without damaging vital organs or vessels becomes very difficult. Compared to other strains, BALB/C mice seem to be more sensitive to IRI-related stress and therefore often recuperate slower or show immediate postoperative mortality.

In summary, we recommend the use of Swiss Webster mice to train junior surgeons or for experiments in which genetic variability is not an issue. C57BL/6 mice should be used when an inbred strain is required or when transgenic or knockout mice are required.

\section{Animal housing and feeding}

As rodents do not regurgitate their food, pre-operative fasting is not necessary. In addition, Verweij et al. (7) described that pre-operative fasting for three days may decrease IRI in mice. We therefore recommend a pre-operative ad libitum laboratory animal diet.

\section{Animal ethics \& welfare}

The Animal Ethics Committee of Antwerp University and the Antwerp-University Hospital-approved our study set-up (file 2012-36). The experimental procedure should include adequate postoperative analgesia to minimize animal discomfort. Buprenorphine was recommended as analgesic agent to relieve postoperative pain by the laboratory animal welfare committee of Antwerp University. Peroperatively, attention should be paid to prevent the corneas from drying out by applying an ophthalmic ointment should be applied to the eyes, to maintain-body temperature needs to be maintained at $36^{\circ} \mathrm{C}$ and continuously monitored by an external heating source and to prevent dehydration by injecting $\underline{A n}$

injection of $0,1 \mathrm{ml}$ of warm sterile isotonic saline subcutaneously before and after surgeryprevents dehydration. Postoperatively, the researcher should actively monitor animal discomfort during 
reperfusion and sacrifice moribund animals according to standard protocols available at the institution's animal ethics board $\underline{(8)} \cdot(8)$

\section{Anesthesia}

The type of anesthesia may influence the outcome of IRI-related experiments(9). (9) The induction agent propofol decreases apoptosis in hepatic IRI and suppresses the inflammatory response after pulmonary IRI. Isoflurane and sevoflurane are known for their attenuative effect on pulmonary IRI, possibly by inhibition of inflammatory factors such as TNF- $\alpha \underline{(10)}$. (10) Xenon anesthesia activates hypoxia-inducible factor $1 \alpha$, protecting the tissue from hypoxic ischemia. Volatile anesthetics are preferred in experimental surgical models, as patients in the clinic are also anesthetized with sevoflurane $\underline{(11) .(11)}$

To induce anesthesia, the animal is placed in an induction chamber, which is connected to an isoflurane vaporizer (IsoTec 3 anesthesia station, GE Healthcare, USA) and exposed to 5\% isoflurane mixed with $95 \%$ oxygen for 4 minutes. The anesthetized animal is taken out of the box and placed on a small hardboard plate (fig 1.12). The front teeth are fixed with an elastic band to the plate (fig 2.1).

\section{Endotracheal intubation and ventilation}

Before intubation, a fiber optic lamp is placed on the skin covering the larynx. The lower jaw is carefully luxated in an upward direction by pulling the tongue out of the mouth with the left index finger and thumb (fig 2.1). Meanwhile, the surgeon softly holds his little fingeffifth digit on the animal's throat. This way, the larynx is stretched and appears as a small, luminous dot at the end of the throat. Oral mucosa or food remnants may obstruct vision and should be removed with blunt forceps. Keeping the larynx in sight, the animal is intubated with a blunt-tipped cannula, fabricated from a 20 G needle of which the sharp tip is carefully filed off (fig 1.19). To verify whether the cannula is correctly placed in the trachea, we use a plastic Pasteur pipette connected with a small tube attached to a Luer lock, which is then coupled to the cannula. When the thoracic cage expands symmetrically after blowing some air through 
the cannula with the pipette, the cannula is in place. If not, the cannula is probably situated in the esophagus and should be replaced.

When the cannula is connected to the ventilator inlet, the thoracic cage should expand synchronically with the ventilator (TOPO Small Animal Ventilator, Kent Scientific, USA). Fixation of the canula with surgical tape ensures that the animal is not accidently extubated, which was the most frequent cause of procedure failure in our lab. Re-intubation is very difficult because of laryngeal edema which occurs during ventilation. Without adequate ventilation, the animal is likely to die once the thoracic cage is opened. After intubation, the induction concentration of $5 \%$ isoflurane is reduced to a maintenance anesthesia of 1.7 to $2 \%$. A continuous anesthesia of $5 \%$ isoflurane results in increased peroperative mortality because of overdosage. If the animal responds to the incision, anesthesia may be insufficient and the isoflurane concentration should be temporarily elevated to 2 to $2.5 \%$.

Using a pressure-guided ventilator, the animal should be ventilated at 120 strokes per minute with a pressure of $17 \mathrm{~cm} \mathrm{H} \mathrm{H}_{2} \mathrm{O}$. Keeping pressure constant during ischemia is important because the total pulmonary volume is significantly reduced as soon as the hilum is occluded. A pressure-controlled ventilator instantly adapts to this volume decrease and keeps intrapulmonary pressure constant at all times, while volume-guided ventilators can only adapt to pressure differences and may overinflate the non-ischemic lung. To avoid this, the original stroke volume of $250 \mu \mathrm{L}$ can be halved to $125 \mu \mathrm{L}$ as soon as the clamp is applied.

Since opening the thoracic cavity annihilates negative intrathoracic pressure, positive end expiratory pressure (PEEP) is required to ensure that the lungs do not collapse. PEEP keeps the lung slightly inflated, even during expiration, to prevent collapse. To achieve this, the exhaust of the ventilator is connected to a PEEP bottle filled with $2 \mathrm{~cm}$ of water. 
When ventilation is optimal, the animal on the hardboard plate is placed on a warm water-heated heating pad. A rectal probe verifies that body core temperature remains constant. We aim to maintain a constant body core temperature of $36^{\circ} \mathrm{C}$. Body temperature is a major cause of variability between procedures and even mild hypothermia may attenuate pulmonary IRI (Shoji Eur Surg Res 2005). Therefore, experiments should be conducted in well-isolated rooms with a more or less controlled temperature. Variations in ambient temperature during reperfusion can also cause variability in IRI.

\section{Animal preparation}

The thorax of the animal is shaved from the armpit to the belly with a razor or a small clipper. The skin is briefly disinfected with iodine solution. The animal is placed with the nose pointing to the right and the tail to the left of the surgeon, with the left flank clearly in sight (fig 2.2). With surgical tape, the left forepaw is fixated in extension towards the intubation cannula. The animal is slightly turned away from the surgeon to expose the complete left flank. This position mimics the preoperative situation of a thoracotomy on human patients. The animal's scapula is palpated and with a sterile marker a line is drawn on the skin from the spinous process of the scapula towards the front of the chest, following the $4^{\text {th }}$ intercostal space.

An injection of $0,2 \mathrm{mg} / \mathrm{kg}$ buprenorphine subcutaneously before incision reduces postoperative animal discomfort.

\section{Surgical procedure}

1. Thoracotomy

Depending on the surgeon's experience, the length of the incision may vary. A larger incision permits better access and a broader surgical field. However, it may also compromise postoperative recovery and may induce postoperative atelectasis when wound closure is insufficient. We advise an incision of about $1 \mathrm{~cm}(0,4$ inch) to maintain good balance between a clear view 
and optimal recovery. The skin incision is made with mayo-style scissors (fig 1.2) and can be elongated with sharp-tipped scissors (fig 1.1). In case of large incisions, special care should be taken to circumvent the lateral thoracic vein that lies in the axilla of the animal. Since this vessel can bleed extensively, it should be ligated or cauterized before any muscles are cut. The subdermal muscle layer is separated using fine-tipped forceps (fig 1.5). To spread the skin borders of the wound, two temporary stitches with a 5.0 silk suture can be made on the upper and lower side of the wound and fixed with tape to the hardboard. This way, the skin flaps and superficial muscle layer can be removed from the operating field. The intercostal muscles, with the movements of the heart and the left lung shining through, are now clearly visible.

Again, the surgeon should look for the $4^{\text {th }}$ intercostal space, which originates at the inferior margin of the scapular spine (fig 2.3). With fine-tipped microsurgical forceps, the $4^{\text {th }}$ intercostal muscle is carefully eroded where the $4^{\text {th }}$ intercostal space crosses the imaginary mid-axillary line. The muscular tissue should be eroded without complete penetration, as this may cause sharp trauma to the underlying lung tissue and air leaks. Subsequently, a small blunt-tipped obtuse forceps (fig 2.4) is pushed through the defect to create a small puncture through the thoracic wall. Carefully pushing through, the forceps should exit again through the $4^{\text {th }}$ intercostal muscle about $0.5 \mathrm{~cm}(0.2 \mathrm{ln})$ from the entrance wound. Because the forceps is blunt, it can be pushed over the lung without damaging the lung underneath. While holding the blunt forceps gently in place, they are now used as a probe over which the intercostal muscle can be safely incised for about $0.5 \mathrm{~cm}(0.2 \mathrm{ln})$ with microsurgical scissors (fig 1.3). The lung is now clearly visible, moving synchronous with the ventilator.

2. Hilar clamping procedure 
A small retractor (No. 17005-03, Fine Science Tools, Germany) (fig 1.18) is placed between the ribs. The thoracic incision can be enlarged to about $1 \mathrm{~cm}(0,4 \mathrm{In})$ if a larger surgical field is necessary for optimal exposure.

Heart and lung lie close to each other in the thoracic cage, separated only by a virtual space. Therefore, the hilum cannot be immediately visualized. The surgeon needs to create a real space between the heart and the lung with small pieces of hemostatic sponge (fig 1.20), which gently push the heart towards the midline and protect the lung from the retractor. The sponges can be picked up with the sharp-tipped microsurgical forceps and bluntly push the heart away to gain a clear view on the hilar structure of the left lung. Through the microscope, the pulmonary artery can be recognized on the right, the pulmonary vein on the left and the bronchus in the middle of the hilum (fig 2.6). With the clamp-applying forceps (fig 1.6), the round clamp (fig 1.7) is opened and placed around the hilum in a fluent, scooping motion to simultaneously occlude the left pulmonary artery, bronchus and pulmonary vein (fig 2.5).

The forceps should be carefully released once the clamp is applied. Instantly, the lung turns whitish instead of pink and become immobile. If respiratory movements caused by the ventilator continue, the clamp is not placed correctly. Placing the clamp is the most difficult part of the procedure and takes some practice. Clearly state the time of ischemia induction.

The retractor is left in place during ischemia. A small cotton swab drained in saline temporarily covers the wound to limit evaporation and prevent tissue and skin to dry out. In our experiments, we maintain ischemia for one hour, during which the animal remains anesthetized using an isoflurane maintenance dose of $1.5 \%$. The surgeon should again pay special attention to minimizing heating loss and monitor temperature during ischemia time. For instance, the animal can be covered with isolating bubble wrap or some other material to keep it warm. In the 
(REFs as in resp to rev EJCTS). Longer ischemia times may compromise animal survival, whereas shorter ischemia times may result in insufficient ischemia and reperfusion injury to the lung to evaluate the effect of different therapeutic strategies.

3. Reperfusion

After one hour, the clamp is carefully removed to allow blood and air flow into the lung, restoring its pink color. Again, state the time when reperfusion commences. The ischemic lung can be deflated and have a blood red instead of pink color. This is characteristic of resorption atelectasis of the lung during ischemia: this is characteristic of ischemia-induced resorption atelectasis, i.e. the clamped lung collapses spontaneously because trapped air slowly diffuses out of the lung. To facilitate full lung expansion, it may be necessary to temporarily increase inspiratory pressure to $20 \mathrm{~cm} \mathrm{H} \mathrm{H}_{2} \mathrm{O}$. As this may cause pressure-induced lung injury, an alternative is to temporary elevate PEEP to $6 \mathrm{~cm} \mathrm{H} 2 \mathrm{O}$ in order to expand the alveoli sufficiently and permits a faster and safer recovery from resorption atelectasis.

4. Wound closure

Closing the intercostal muscles with a 5.0 polypropylene suture requires special attention to avoid damage to the underlying lung tissue. Two to three sutures with a simple double knot should suffice to reduce any air leak. The suture, as in human thoracotomy, should approximate the $4^{\text {th }}$ and $5^{\text {th }}$ ribs closely together (fig 2.7). The superficial muscle layer is then sutured with a 4.0 silk thread. To evaluate the airtightness of the wound closure, a drop of saline is applied on the wound. Air bubbles indicate imperfect sutures and demand a close 
inspection of the knots and sutures. Finally, skin sutures with 4.0 silk suture should neatly close the wound, without any defects.

\section{Postoperative care}

The isoflurane concentration is reduced to zero, but the ventilation with $95 \%$ oxygen should be continued for about 5 minutes. If the animal is clearly in pain despite the buprenorphine injection, another subcutaneous injection can be given. Note, however, that buprenorphine is more effective if administered before the procedure. It can also reach a ceiling effect, when the analgesic effect of the drug no longer linearly increases with the dosage. A decent alternative is subcutaneous morphine (10 $\mathrm{mg} / \mathrm{kg})(12)$, which is a fast-acting analgesic. Placing the mouse with its forepaws on a roll of cotton swabs (fig 2.8) takes away mechanic pressure from the thoracic cage and maximizes breathing comfort for the animal. If the larynx was injured during intubation, laryngeal edema may occur postoperatively. Therefore the animal might not be able to breathe sufficiently by itself immediately after the procedure. Re-intubation after weaning from the ventilator is very difficult because of edema and can compromise animal survival postoperatively. To prevent this, the tracheal cannula should be left in place as long as the animal is not responding. Supportive ventilation and oxygenation improves outcome significantly. When the animal shows signs of increased activity, the cannula should be immediately removed. The recovered animal is placed in a clean cage with easy access to water and chow and a comfortable animal shelter. An injection of $0,5 \mathrm{ml}$ of saline subcutaneously counters insensible water loss during the procedure. The animals should be kept warm with an ultraviolet heat lamp placed on sufficient distance to avoid hyperthermia or heat burns.

When the appropriate reperfusion time has been reached, the animal can be sacrificed and any organ of interest recovered for later analysis. Adequate methods of sacrificing the animals are cervical dislocation 
or controlled exsanguination through vena cava posterior after laparotomy during a short anesthesia (13). $(13)$

\section{Evaluation of the ischemia-reperfusion injury murine model}

Histological analysis is still the golden standard to evaluate tissue injury resulting from ischemia and reperfusion. In order to affirm that the model we present in this paper is valuable, we have conducted a pilot experiment in which 5 Swiss/Webster mice were subjected to a sham operation (thoracotomy only), $1 \mathrm{~h}$ of ischemia alone, and $1 \mathrm{~h}$ of ischemia followed by $1 \mathrm{~h}, 4 \mathrm{~h}$ or $24 \mathrm{~h}$ of reperfusion. After the given time, the animals were sacrificed by exsanguination. Left lungs were briefly rinsed in cold PBS, fixated for 2 hours in a $4 \%$ formaldehyde solution, embedded in paraffin and subsequently cut in $5 \mu \mathrm{m}$ sections for histological analysis. Lymphocyte detection with immunohistochemistry was performed using a rabbit polyclonal to $\operatorname{CD} 3$ (Abcam) according to manufacturer's instruction. Secondary antibody bindings (alkalic phosphatase) was visualized with a BCIP/NBT Substrate System (DAKO). The amount of apoptotic cells is determined by immunohistochemistry with an antibody against cleaved caspase 3 (Cell Signalling Technology). Histological analysis was performed by a blinded pathologist (JPB) who had no knowledge of the experimental design. Extent of IRI was evaluated on criteria derived from the literature, such as lymphocyte infiltration (SLI), neutrophil recruitment (NE), alveolar edema (AE), edema of the alveolar septa (SE) and the occurrence of hyaline membranes (HM). The presence of each criterium was graded as absent $(-)$, mild $( \pm)$, clearly present $(+)$ or intense $(++)$. Results are presented in table 2 and figure 3. The number of infiltrated lymphocytes, recruited neutrophils and apoptotic nuclei was determined per five high power fields (HPF) in 15 mice ( $n=5$ per IR group). Statistical analysis was performed using GraphPad Prism v.6 (Graphpad, USA) with one-way analysis of variance and significance assumed at $\mathrm{p}<0,05$ with least significant difference (LSD) as post-hoc test. 


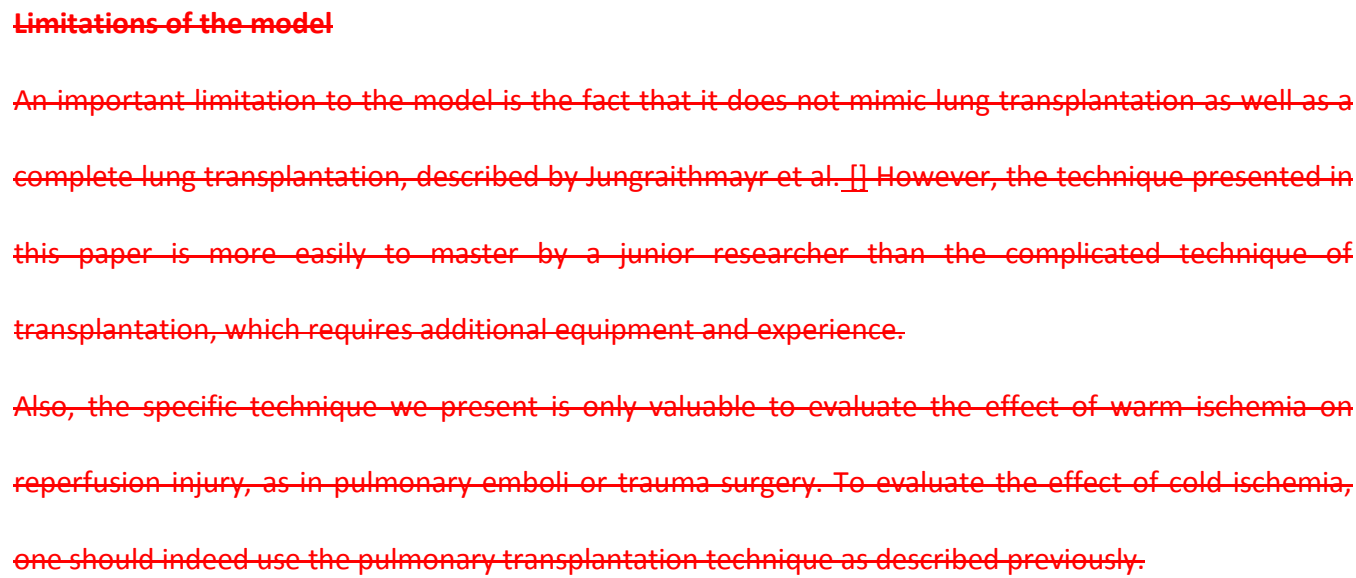

\section{Conclusion}

IRI is a complex process with major clinical implications, for instance in transplantation surgery. Research into the pathogenesis and possible therapies of pulmonary IRI can counter postoperative morbidity and mortality following thoracic surgery. Laboratory animal models of pulmonary IRI are available, but involve a challenging surgical procedure. Our paper provides a detailed, illustrated manual with tips and tricks to guide even an apprentice surgeon to master this technique and obtain reliable results with minimal mortality.

\section{Grants}

This project was funded by a research fellowship (aspirant) awarded to Jan F. Gielis by the Research Foundation - Flanders (FWO [Grant no. 11F6513N] 


\section{Acknowledgements}

The authors thank Annemie Van Den Broeck, Joke De Raedemaecker, Sofie Thys and Martine Van den Broeck for their excellent technical assistance. 


\section{Figure legends}

\section{Figure 1 : Necessary surgical tools and materials (summed up in table 1).}

\section{Table 1: Necessary surgical tools and materials}

\section{Figure 2: Different stages of the surgical procedure}

(1) the animal is intubated with a blunt needle while placing a light source on the laryngeal region; (2) the animal is fixated and the left flank and back are exposed to the surgeon; (3) after skin incision the shoulder and pectoral muscles are retracted; identification of 4th intercostal muscle; (4) using blunt curved forceps the intracostal muscles are pierced and transected; (5) and (6) an atraumatic clamp is placed on the pulmonary hilum; (7) careful closing of the wound prevents air-leak; (8) post-operative recovery minimizing breathing effort.

\section{Table 2: Histologic evaluation of ischemia-reperfusion injury}

(SLI, septal lymphocyte infiltration; $A E$, alveolar edema; SE, septal edema; NE, neutrophil infiltration; $A B$, alveolar bleeding; HM, hyaline membranes).

\section{Figure 3: lung histology during ischemia-reperfusion injury}

From top to bottom: evaluation of lymphocyte infiltration by CD3-immunohistochemistry; haematoxyllin-eosin (HE) stain with arrows indicating lymphocyte aggregations (thin black arrows) after 1h of reperfusion, neutrophil nests (thick gray arrows) and alveolar edema (thick black arrows) after 4 hours of reperfusion; activated caspase 3 (ActCasp3) immunohistochemistry shows occurrence of apoptosis after 4 hours of reperfusion. Magnification is indicated for each slide. (I, ischemia; R, reperfusion; $\mathrm{HE}$, haematoxyllin-eosin)

\section{Figure 4: Evolution of cellular infiltration and apoptosis during ischemia-reperfusion injury}

Lymphocyte infiltration, neutrophil recruitment and apoptosis were counted in five high-power fields per lung section. $n=5$ animals per group. We observed a large increase in lymphocyte infiltration after 1 hour of reperfusion which normalized after 4 hours, and recruitment of neutrophils which increased during reperfusion. The amount of apoptotic cells was only increased after 4 hours of reperfusion. ${ }^{*} p<0,05$, $* * p<0,005$. Data show mean \pm SEM, two-way ANOVA with Least Significant Difference (LSD) post-hoc (I, ischemia; R, reperfusion; actCasp3, activated caspase 3; IRI, ischemia-reperfusion injury; HPF, high power field). 


\section{References}

1. Matute-Bello G, Frevert CW, Martin TR Animal models of acute lung injury. American journal of physiology. Lung cellular and molecular physiology 2008:295:L379-399.

2. Land $\mathrm{W}$, Meßmer $\mathrm{K}$ The impact of ischemia-reperfusion injury on specific and non-specific, early and late chronic events after organ transplantation. In: Touraine JL, Traeger J, Bétuel H, Dubernard JM, Revillard JP, et al. eds., Organ AllocationSpringer Netherlands, 1998:233-260.

3. de Perrot M, Liu M, Waddell TK, Keshavjee $S$ Ischemia-reperfusion-induced lung injury. American journal of respiratory and critical care medicine 2003:167:490-511.

4. Christie JD, Kotloff RM, Pochettino A, Arcasoy SM, Rosengard BR, et al. Clinical risk factors for primary graft failure following lung transplantation. CHEST Journal 2003:124:1232-1241.

5. Burne MJ, Haq M, Matsuse H, Mohapatra S, Rabb H Genetic susceptibility to renal ischemia reperfusion injury revealed in a murine model. Transplantation 2000:69:1023-1025.

6. van den Borne SW, van de Schans VA, Strzelecka AE, Vervoort-Peters HT, Lijnen PM, et al. Mouse strain determines the outcome of wound healing after myocardial infarction. Cardiovascular research 2009:84:273-282.

7. Verweij M, van Ginhoven TM, Mitchell JR, Sluiter W, Den Engel SV, et al. Preoperative fasting protects mice against hepatic ischemia/reperfusion injury: mechanisms and effects on liver regeneration. Liver transplantation 2011:17:695-704.

8. Wolfensohn S, Lloyd M Handbook of laboratory animal management and welfare. John Wiley \& Sons, 2013.

9. Eroglu A The effect of intravenous anesthetics on ischemia-reperfusion injury. BioMed research international 2014:2014:821513.

10. Liu $\mathrm{R}$, Ishibe $\mathrm{Y}$, Ueda $\mathrm{M}$ Isoflurane-sevoflurane adminstration before ischemia attenuates ischemia-reperfusion-induced injury in isolated rat lungs. Anesthesiology 2000:92:833-840.

11. Erturk E Ischemia-reperfusion injury and volatile anesthetics. BioMed research international 2014:2014:526301.

12. Miller AL, Richardson CA Rodent analgesia. The veterinary clinics of North America. Exotic animal practice 2011:14:81-92.

13. Parasuraman S, Raveendran R, Kesavan R Blood sample collection in small laboratory animals. Journal of pharmacology \& pharmacotherapeutics 2010:1:87-93. 